\title{
Overexpression of IL-32 is a novel prognostic factor in patients with localized clear cell renal cell carcinoma
}

\author{
HYUN-JUNG LEE ${ }^{1 *}$, ZHE LONG LIANG ${ }^{1 *}$, SONG MEI HUANG $^{1}$, \\ JAE-SUNG LIM ${ }^{2}$, DO-YOUNG YOON ${ }^{3}$, HYO-JIN LEE ${ }^{4}$ and JIN MAN KIM ${ }^{1,5}$
}

\begin{abstract}
Departments of ${ }^{1}$ Pathology and ${ }^{2}$ Urology, Chungnam National University School of Medicine, Daejeon;
${ }^{3}$ Department of Bioscience and Biotechnology, Bio/Molecular Informatics Center, Konkuk University, Seoul; ${ }^{4}$ Department of Internal Medicine, Chungnam National University Hospital; ${ }^{5}$ Regional Cancer Center and Infection Signaling Network Research Center, Chungnam National University School of Medicine, Daejeon, Republic of Korea
\end{abstract}

Received July 19, 2011; Accepted November 3, 2011

DOI: $10.3892 / \mathrm{ol} .2011 .511$

\begin{abstract}
Interleukin-32 (IL-32) is a proinflammatory cytokine that acts as a significant pathogenetic factor in various diseases and malignancies. However, the clinical effect of IL-32 expression in renal cell carcinoma ( $\mathrm{RCC}$ ) has not previously been investigated. The aim of the present study was to examine the significance of IL-32 overexpression in localized clear cell RCC (CCRCC). We examined 112 patients with localized CCRCC who underwent nephrectomy. The clinicopathological data were obtained by retrospective review and the expression levels of IL-32 were studied by immunohistochemistry. Tumors were classified according to staining intensity ( 0 , no staining intensity; 1 , weak; 2 , intermediate; 3 , strong). The cases with staining intensities from 0 to 2 comprised the IL-32 low-expression group (LEG), whereas those with a staining intensity of 3 comprised the IL-32 high-expression group (HEG). Correlations between IL-32 expression and clinicopathological parameters were determined. Staining intensities were determined for all cases as follows: 26 cases (23.2\%) (score 0), 43 cases (38.4\%) (score 1), 31 cases $(27.7 \%$ ) (score 2) and 12 cases (10.7\%) (score 3). IL-32 HEG exhibited a higher recurrence rate compared to the IL-32 LEG (50 vs. 13\%, $\mathrm{P}=0.001)$. For survival rates, the 5-year recurrence-free survival (RFS), disease-specific survival (DSS) and overall survival (OS) rates were lower in the IL-32 HEG group compared with the IL-32 LEG group (RFS, $\mathrm{P}=0.001$; DSS, $\mathrm{P}<0.001$;
\end{abstract}

Correspondence to: Professor Hyo-Jin Lee, Department of Internal Medicine, Chungnam National University Hospital, 640 Daesa-dong, Jung-gu, Daejeon, Republic of Korea

E-mail: cymed@cnu.ac.kr

Professor Jin Man Kim, Department of Pathology and Regional Cancer Center and Infection Signaling Network Research Center, Chungnam National University School of Medicine, Daejeon, Republic of Korea

\section{"Contributed equally}

Key words: interleukin-32, renal cell carcinoma, prognosis
OS, $\mathrm{P}=0.026$, respectively). Univariate analyses revealed that Fuhrman nuclear grade and a high IL-32 expression were significant prognostic factors for predicting RFS, DSS and OS in CCRCC, whereas multivariate analyses indicated that Fuhrman nuclear grade and high IL-32 expression were still independent risk factors. In conclusion, IL-32 overexpression was associated with high recurrence rates and low RFS, DSS and OS, indicating that it may be a novel prognostic factor for predicting outcomes in patients with CCRCC.

\section{Introduction}

Renal cell carcinoma (RCC) is the most common primary malignant neoplasm of the kidney and one of the most fatal genitourinary malignancies. The incidence is consistently $3 \%$ of all malignant neoplasms and the lethality from RCC accounts for $4 \%$ of all deaths from malignancies (1). Even following curative nephrectomy, approximately $20 \%$ of RCC patients experience disease recurrence within 5 years (2). Following metastasis, RCC exhibits markedly poor prognosis, although immunotherapy and several targeted agents improve the survival in certain populations (3). It is therefore crucial to predict which patients are likely to develop disease recurrence after surgery for localized RCC.

The prognostic factors of non-metastatic RCC have been studied using various methods. Traditionally, the tumor-nodemetastasis (TNM) stage, Fuhrman nuclear grade, Eastern Cooperative Oncology Group performance status (ECOG PS), and tumor necrosis were determined to be classic clinicopathologic prognostic factors (4). In molecular studies, biomarkers such as vascular endothelial growth factor (VEGF) (5), p53 mutation (6), hypoxia-inducible factor $1 \alpha$ (7), and C-reactive protein (8) were identified as effective prognostic factors. In genetics, the Von-Hippel-Lindau (VHL) gene was thought to be associated with a more favorable prognosis than the sporadic form (9). However, the majority of molecular and genetic factors do not have notable impact significance in RCC.

Interleukin-32 (IL-32), previously denoted as natural killer cell transcript 4 (NK4), was first identified as a transcript in IL-2-activated natural killer (NK) cells and T lymphocytes in 1992 by Dahl et al (10). IL-32 is now recognized as a new 
proinflammatory cytokine produced by epithelial cells and monocytes, as well as NK cells and T lymphocytes (11). The encoding gene of IL-32 is located at chromosome 16p13.3 and six splice variants have been reported: IL-32 $\alpha$, IL-32 $\beta$, IL-32 $\gamma$, IL-32 $\delta$, IL-32 $\varepsilon$, and IL-32 $\xi$ (12). One study identified the specific receptor of IL-32 as proteinase-3 (13). The exact function of IL-32 is not clear but it has been implicated in inflammatory disorders such as rheumatoid arthritis (14), Mycobacterium tuberculosis infection (15), and inflammatory bowel disease (16). Recently, there has been a focus on the expression of IL-32 in human malignancies such as stomach cancer (17), lung cancer (18), pancreatic cancer (19), hematopoietic malignancies $(20,21)$, and uterine cervical cancer (22). There are no previous studies about the expression of IL-32 in RCC. The aim of the present study was to investigate IL-32 expression in CCRCC tissue and reveal the novel value of IL-32 as a prognostic factor.

\section{Materials and methods}

Patients and tumor samples. We investigated 112 consecutive patients with CCRCC who underwent radical or partial nephrectomy for sporadic, localized (T1-3N0M0) RCC at the Chungnam National University Hospital, Korea, between 1999 and 2006. Clinicopathologic data of patients were obtained by reviewing medical records. ECOG performance status was assigned to each patient at the time of diagnosis. $\mathrm{T}$ classification was defined according to the 2002 American Joint Committee on Cancer criteria and the nuclear grade according to the Fuhrman grading system. Tumor samples were collected from the tissue blocks used for routine pathologic examination. All patients signed informed consent for therapy as well as for subsequent tissue studies, which had received prior approval by the local ethics committee.

Tissue microarray construction. Tissue microarrays were constructed from archival original formalin-fixed, paraffinembedded tissue blocks from 112 cases of localized CCRCC. For each tumor, a representative tumor area was carefully selected from a hematoxylin and eosin-stained section of a donor block. Each case was represented by 2 cores of 2-mm cylinders from tumors that were punched using an automated tissue array (UNITMA, Seoul, Korea). Thus, tissue microarray blocks containing 224 cylinders were constructed.

Specimen preparation and immunohistochemistry. For immunohistochemistry, sections $(3-\mu \mathrm{m})$ were cut from the recipient blocks and placed on 3-amino-propyltriethoxysilane-coated slides that were dried for $2 \mathrm{~h}$ prior to staining at $57^{\circ} \mathrm{C}$. Procedures were performed at room temperature, as recommended by the manufacturer. In brief, sections were dewaxed in xylene and rehydrated in graded alcohols. Sections were then washed in water prior to antigen retrieval, using a Dako PTLink machine (Dako, Glostrup, Denmark) with $10 \mathrm{mM}$ sodium citrate buffer $(\mathrm{pH} 6.0)$ at $97^{\circ} \mathrm{C}$ for 20 min. Sections were treated with $3 \%$ hydrogen peroxide for 10 min to block endogenous peroxidase and were then preincubated with a serum-free protein block solution (Dako, Carpinteria, CA, USA) for $20 \mathrm{~min}$ to eliminate background staining. A monoclonal mouse antibody against human IL-32 was used, as previously described (11). The slides were then incubated for $30 \mathrm{~min}$ with an EnVision anti-mouse (Dako, Denmark) polymer. Reaction products were visualized with diaminobenzidine plus substrate-chromogen solution applied for $5 \mathrm{~min}$. The slides were counterstained with Meyer's hematoxylin and mounted. The sections were carefully rinsed with several changes of phosphate-buffered saline between each stage of the procedure. Negative controls were yielded by excluding the primary antibody or by using preimmune IgG1 to evaluate non-specific staining.

Evaluation of immunohistochemical staining. The results yielded by immunohistochemical staining were evaluated by two independent pathologists (J.M. Kim and H.J. Lee) who were blinded to the clinicopathological data of the patients. Immunohistochemical staining was classified according to a scoring method; tumors were classified into four grades based on the staining intensity (score 0 , no staining intensity; score 1, weak staining intensity; score 2, intermediate staining intensity; score 3, strong staining intensity). In cases of heterogeneous staining within the samples, the respective higher score was selected when $>50 \%$ of the cells exhibited higher staining intensity. For all patients, scores from two tumor cores from the same patient were averaged to obtain a mean score. The cases with staining intensity scores of 0 , 1, and 2 comprised the IL-32 low-expression group (LEG), whereas those with score 3 staining intensity comprised the IL-32 high-expression group (HEG).

Statistical analysis. Pearson's Chi-square test was used to assess the associations between the expression of IL-32 and the clinicopathological parameters. To estimate the 5-year recurrence-free survival (5yr-RFS), 5-year disease-specific survival (5yr-DSS), and 5-year overall survival (5yr-OS) rates, we used the Kaplan-Meier method and log-rank test. The 5yr-RFS was measured from the date of nephrectomy to the date of recurrence or death from RCC (5yr-RFS). The 5yr-DSS was measured from the date of nephrectomy to the date of death from RCC only. The 5yr-OS was measured from the date of surgery to the date of death from any causes. To analyze the effect of the expression of IL-32 on RFS, DSS and OS, Cox's proportional hazards model was used. For all analyses, $\mathrm{P}<0.05$ was considered statistically significant. Statistical analyses were conducted using the SPSS version 13.0 statistical software program (SPSS Inc., Chicago, IL, USA).

\section{Results}

Patient characteristics. The median age of the patients was 60 years (range 30-78), with a male predominance (male:female ratio of 2.4:1). ECOG 0 patients comprised $43.8 \%$ of the group and the remaining patients $(56.3 \%)$ were in other ECOG performance-status groups $(\geq 1)$. The tumor sizes varied from 1 to $15 \mathrm{~cm}$ at the largest diameter and the median size was $5 \mathrm{~cm}$. Of the 112 patients, 41.9, 16.1 and $42.0 \%$ were found to have $\mathrm{pT} 1, \mathrm{pT} 2$ and $\mathrm{pT} 3$ primary tumors, respectively. The Fuhrman nuclear grade revealed grade $1,2,3$ and 4 lesions in 15.2, 67.9, 14.3 and $2.7 \%$ of patients, respectively. Nineteen patients $(17 \%)$ experienced recurrence following nephrectomy. 


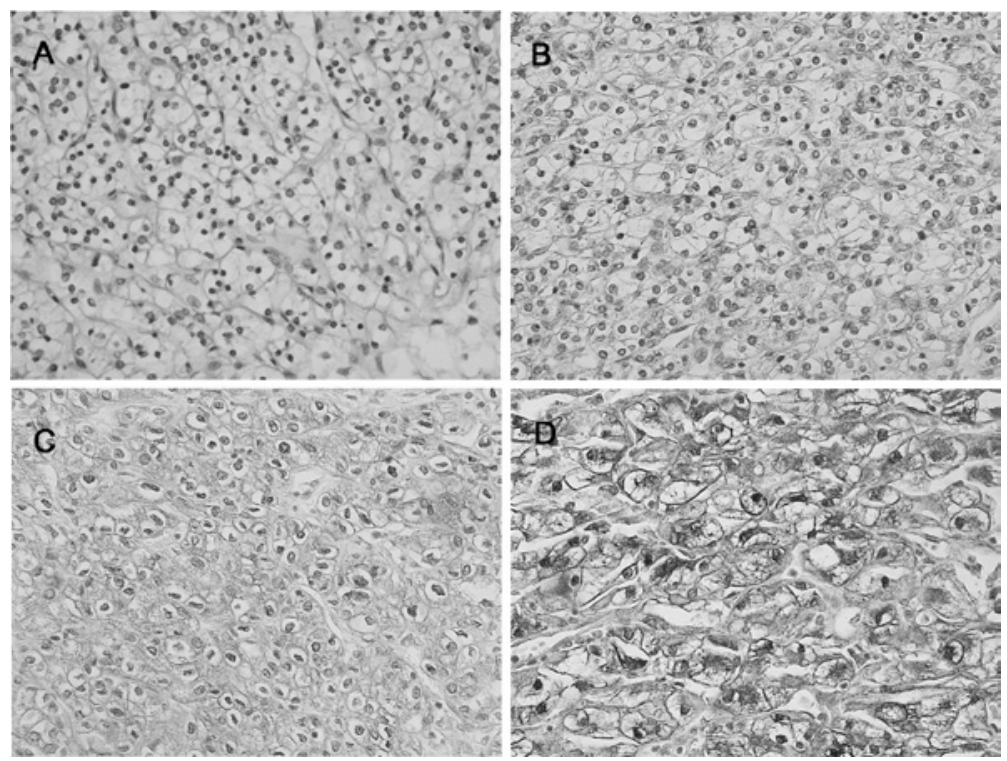

Figure 1. Representative photomicrographs of immunohistochemical staining for IL-32 in renal cell carcinoma tissues. (A) No staining intensity, (B) weak staining intensity, (C) intermediate staining intensity and (D) strong staining intensity (magnification, x400).

Immunohistochemical staining for IL-32 expression in $C C R C C$. In the majority of cases, the cytoplasm of the neoplastic cells stained positive for IL-32, and certain cells also stained in the nucleus and cytoplasmic membrane (Fig. 1). We estimated the level of IL-32 expression in the positively stained tumor cells using a staining intensity score of $0,1,2$ or 3 . In $26(23.2 \%)$ of the 112 cases, the tumor cells did not exhibit IL-32 expression and 43 cases (38.4\%) exhibited weak positivity (staining intensity score 1$)$. Intermediate staining was found in 31 cases $(27.7 \%$ ) (score 2 ) and 12 cases $(10.7 \%)$ showed a marked staining intensity (score 3 ). We divided the cases into two groups: Cases with scores from 0 to 2 comprised the IL-32 low-expression group (LEG) and score 3 cases comprised the IL-32 high-expression group (HEG). Therefore, 100 cases were classified into the IL-32 LEG while 12 cases were classified into the IL-32 HEG (Table I).

Association between IL-32 expression and clinicopathological characteristics. Analysis of the association between IL-32 expression and clinicopathologic characteristics showed a significant difference in the recurrence rate $(\mathrm{P}=0.001)$ (Table I). In the IL-32 LEG, recurrence was found in 13 cases (13\%) following nephrectomy, whereas recurrence was noted in 6 cases $(50 \%)$ in the IL-32 HEG. No significant differences were observed in age, gender, ECOG PS, tumor size, T stage, and Fuhrman nuclear grade between the IL-32 LEG and IL-32 HEG groups.

Correlation between IL-32 expression and survival. To determine the benefits of IL-32 expression in CCRCC, we investigated the associations between IL-32 expression and RFS, DSS and OS. The 5-year RFS, DSS and OS rates were $83.0,88.4$ and $81.3 \%$, respectively, for the whole study population. The IL-32 LEG was censored 1 and the HEG was censored 2. The 5yr-RFS rates of the IL-32 LEG and HEG groups were 87 and 50\%, respectively (Fig. 2A; P=0.001), and the rates of 5yr-DSS for the IL-32 LEG and HEG
Table I. Correlation between the expression of IL-32 and clinicopathological parameters.

\begin{tabular}{|c|c|c|c|c|c|}
\hline \multirow[b]{3}{*}{ Characteristic } & \multicolumn{4}{|c|}{ IL-32 expression } & \multirow[b]{3}{*}{$\mathrm{P}$} \\
\hline & \multicolumn{2}{|c|}{$\begin{array}{c}\text { LEG } \\
(n=100)\end{array}$} & \multicolumn{2}{|c|}{$\begin{array}{l}\text { HEG } \\
(n=12)\end{array}$} & \\
\hline & No. & $\%$ & No. & $\%$ & \\
\hline \multicolumn{6}{|l|}{ Age (years) } \\
\hline$\leq 70$ & 85 & 85.0 & 8 & 66.7 & 0.110 \\
\hline$>70$ & 15 & 15.0 & 4 & 33.3 & \\
\hline \multicolumn{6}{|l|}{ Gender } \\
\hline Male & 69 & 69.0 & 10 & 83.3 & 0.303 \\
\hline Female & 31 & 31.0 & 2 & 16.7 & \\
\hline \multicolumn{6}{|l|}{ ECOG PS } \\
\hline 0 & 41 & 41.0 & 8 & 66.7 & 0.090 \\
\hline$\geq 1$ & 59 & 59.0 & 4 & 33.3 & \\
\hline \multicolumn{6}{|c|}{ Tumor size $(\mathrm{cm})$} \\
\hline$\leq 10$ & 94 & 94.0 & 11 & 91.7 & 0.752 \\
\hline$>10$ & 6 & 6.0 & 1 & 8.3 & \\
\hline \multicolumn{6}{|l|}{$\mathrm{T}$ stage } \\
\hline $\mathrm{T} 1$ & 59 & 59.0 & 6 & 50.0 & 0.551 \\
\hline $\mathrm{T} 2 / 3$ & 41 & 41.0 & 6 & 50.0 & \\
\hline \multicolumn{6}{|l|}{$\begin{array}{l}\text { Fuhrman } \\
\text { nuclear grade }\end{array}$} \\
\hline $\mathrm{G} 1 / 2$ & 83 & 83.0 & 10 & 83.3 & 0.977 \\
\hline $\mathrm{G} 3 / 4$ & 17 & 17.0 & 2 & 16.7 & \\
\hline \multicolumn{6}{|l|}{ Recurrence } \\
\hline No & 87 & 87.0 & 6 & 50.0 & 0.001 \\
\hline Yes & 13 & 13.0 & 6 & 50.0 & \\
\hline
\end{tabular}

ECOG, Eastern Cooperative Oncology Group; PS, performance status; LEG, low-expression group; HEG, high-expression group. 

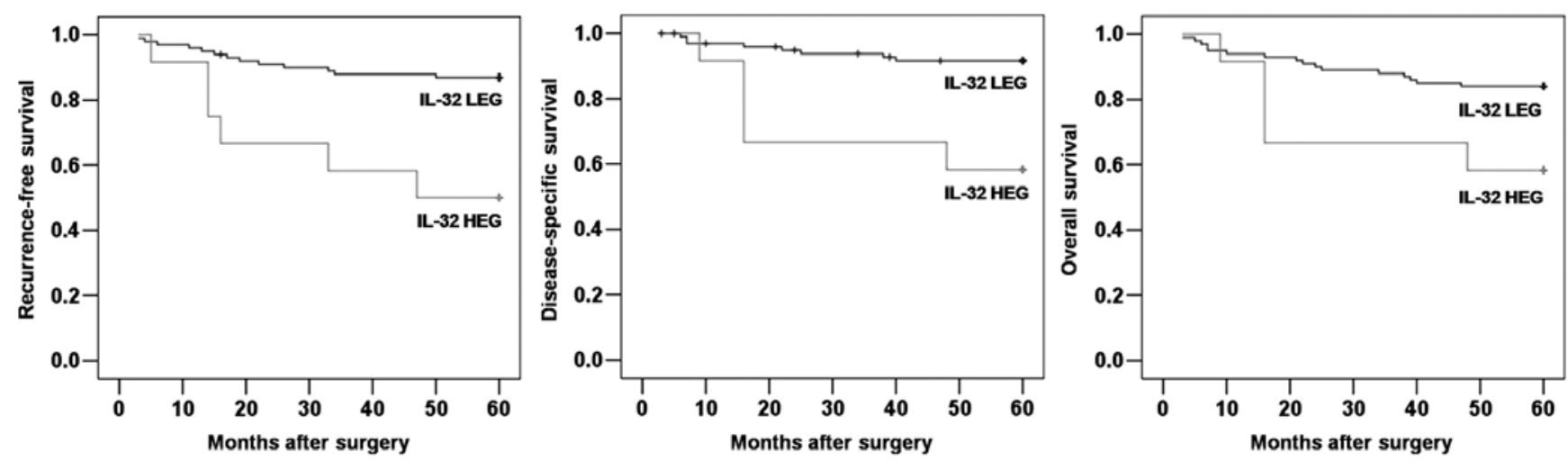

Figure 2. Correlation between IL-32 expression and survival rates in 112 patients with clear cell RCC. (A) 5yr-RFS (P=0.001), (B) 5yr-DFS (P<0.001) and (C) $5 y r-O S(P=0.026)$.

groups were 92 and 58.3\%, respectively (Fig. 2B; P<0.001). The 5yr-OS rates for the two groups were $84 \%$ and $58.3 \%$, respectively (Fig. 2C; $\mathrm{P}=0.026$ ). Therefore, the 5-year RFS, DSS, and OS of the IL-32 LEG group were significantly higher than those of the IL-32 HEG group.

To determine the clinical significance of various parameters that may affect tumor recurrence and survival in CCRCC, we performed univariate analyses (Table II). The Fuhrman nuclear grade and a high IL-32 expression were significant risk factors affecting RFS ( $\mathrm{P}=0.009$ and $\mathrm{P}=0.002$, respectively). For DSS and OS, the Fuhrman nuclear grade $(\mathrm{P}=0.005$ and $\mathrm{P}=0.026$, respectively) and high IL-32 expression $(\mathrm{P}=0.002$ and $\mathrm{P}=0.034$, respectively) showed statistical significance.

To determine the independent prognostic factors, multivariate analyses were then performed using Cox's proportional hazards model (Table III). The model revealed that Fuhrman nuclear grade [hazard ratio (HR), 3.052; 95\% confidence interval (95\% CI), 1.075-8.664; $\mathrm{P}=0.036$ ] and a high IL-32 expression (HR, 4.932; 95\% CI, 1.781-13.657; $\mathrm{P}=0.009$ ) were independent risk factors of RFS in CCRCC patients. With regard to predicting the DSS and OS, the Fuhrman nuclear grade and a high IL-32 expression were also significant as independent prognostic factors. For DSS, the Fuhrman nuclear grade HR was 4.688 (95\% CI, 1.389 15.814; $\mathrm{P}=0.013)$ and the high IL-32 expression HR was 6.736 (95\% CI, 2.01-22.563; $\mathrm{P}=0.002)$. For OS, the Fuhrman nuclear grade HR was 2.741 (95\% CI, 1.056-7.114; $\mathrm{P}=0.038$ ) and the high IL-32 expression HR was 2.992 (95\% CI, 1.066-8.394; $\mathrm{P}=0.037$ ). Moreover, the results revealed that a high expression of IL-32 in CCRCC was a more significant prognostic factor for predicting the RFS, DSS and OS than the Fuhrman nuclear grade (Table III).

\section{Discussion}

IL-32, initially denoted as NK4, was identified as a transcript produced by mitogen-activated T-cells and IL-2-activated NK-cells. Dahl et al, the first group to report NK4 in 1992, proposed that NK4 was a novel common product of activated NK and $\mathrm{T}$ cells that showed no homology with other sequences (10). Further studies of NK4 termed it IL-32 following the detection of cytokine-like gene expression in an IL-18-induced microarray (11). The IL-32 encoding gene locus has been identified at chromosome 16p13.3 and six

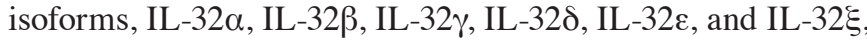
were identified by Kim et al and Chen et al $(11,12)$. Moreover, several studies identified the production of IL-32 in other cell types, including epithelial cells and other monocytes, as well as the presence of IL-32 mRNA in Epstein-Barr virus-infected lymphoma, neuroblastoma, and hematopoietic progenitor cells (23-25).

IL-32 has been evaluated as an entity in a variety of diseases. Initially, the focus was on the expression of IL-32 in inflammatory diseases such as tuberculosis, rheumatoid arthritis, and inflammatory bowel disease. IL-32 pathways are reportedly involved in immune response. Initially, it was determined that IL-32 activated the NF- $\mathrm{KB}$ and p38MAPK signaling pathways (11). Netea et al demonstrated the synergetic effects of IL-32 with the nucleotide oligomerization domain (NOD) 1 and NOD 2 ligands through a caspase-1-dependent mechanism (26). Moreover, findings of recent studies have shown that IL-32 is involved in cancer pathogenesis. Elevated serum IL-32 levels were detected in stomach cancer patients and secreted IL-32 was increased in a K-562 lymphoblastic cell line as detected by ELISA and immunohistochemistry (17). Nishida et al demonstrated IL-32 expression in human pancreatic tissue and pancreatic cancer cell lines. These authors showed that although normal pancreatic duct cells exhibited weak positive staining, the staining intensity was markedly increased in chronic pancreatitis and strong in pancreatic cancer cells. This study was based on the hypothesis that chronic pancreatitis was a precancerous condition (19). Sorrentino et al studied the expression of IL-32 in human lung cancer (18). These authors demonstrated that malignancies expressed IL-32 with marked positive reactivity by immunohistochemical staining, with the exception of squamous cell carcinomas. They also focused on the relationship between inflammation and lung cancer and proposed that the proinflammatory cytokine IL-32 is involved in lung carcinogenesis (18). However, no previous study examined the clinicopathological significance between IL-32 expression and RCC.

RCC is the most common and lethal malignancy of the genitourinary tract cancers (1). Five subtypes are recognized: clear cell, papillary, chromophobe, collecting duct, and unclassified renal cell carcinoma (27). The most common subtype is CCRCC, which represents up to $82 \%$ of all renal carcinomas. Various prognostic factors, particularly in localized renal cell 
Table II. Univariate analyses of clinicopathological parameters and IL-32 expression and their association with prognosis in clear cell renal cell carcinoma.

\begin{tabular}{|c|c|c|c|}
\hline Variables & Hazard ratio & $\begin{array}{l}95 \% \text { Confidence } \\
\text { interval }\end{array}$ & $\mathrm{P}$ \\
\hline \multicolumn{4}{|l|}{ Recurrence-free survival } \\
\hline ECOG PS $(\geq 1 / 0)$ & 0.858 & $0.349-2.111$ & 0.739 \\
\hline Tumor size $(>10 / \leq 10 \mathrm{~cm})$ & 3.440 & $1.000-11.836$ & 0.050 \\
\hline Pathological T stage (T2-3/T1) & 2.386 & $0.859-6.627$ & 0.095 \\
\hline Fuhrman nuclear grade (G3-4/G1-2) & 3.472 & $1.366-8.827$ & 0.009 \\
\hline IL-32 expression (HEG/LEG) & 4.694 & $1.179-12.384$ & 0.002 \\
\hline \multicolumn{4}{|l|}{ Disease-specific survival } \\
\hline ECOG PS $(\geq 1 / 0)$ & 0.895 & $0.301-2.664$ & 0.842 \\
\hline Tumor size $(>10 / \leq 10 \mathrm{~cm})$ & 3.026 & $0.670-13.661$ & 0.150 \\
\hline Pathological T stage (T2-3/T1) & 2.803 & $0.771-10.189$ & 0.117 \\
\hline Fuhrman nuclear grade (G3-4/G1-2) & 4.737 & $1.590-14.117$ & 0.005 \\
\hline IL-32 expression (HEG/LEG) & 5.766 & $1.882-17.671$ & 0.002 \\
\hline \multicolumn{4}{|l|}{ Overall survival } \\
\hline ECOG PS $(\geq 1 / 0)$ & 0.840 & $0.357-1.977$ & 0.689 \\
\hline Tumor size $(>10 / \leq 10 \mathrm{~cm})$ & 1.773 & $0.413-7.614$ & 0.441 \\
\hline Pathological T stage (T2-3/T1) & 1.688 & $0.681-4.185$ & 0.258 \\
\hline Fuhrman nuclear grade (G3-4/G1-2) & 2.799 & $1.128-6.943$ & 0.026 \\
\hline IL-32 expression (HEG/LEG) & 2.962 & $1.083-8.101$ & 0.034 \\
\hline
\end{tabular}

ECOG, Eastern Cooperative Oncology Group; PS, performance status; LEG, low-expression group; HEG, high-expression group.

Table III. Multivariate analyses of clinicopathological parameters and IL-32 expression and their association with prognosis in clear cell renal cell carcinoma.

\begin{tabular}{|c|c|c|c|}
\hline Variables & Hazard ratio & $95 \%$ confidence interval & $\mathrm{P}$ \\
\hline \multicolumn{4}{|l|}{ Recurrence-free survival } \\
\hline ECOG PS $(\geq 1 / 0)$ & 1.043 & $0.395-2.750$ & 0.933 \\
\hline Tumor size $(>10 / \leq 10 \mathrm{~cm})$ & 1.620 & $0.396-6.625$ & 0.502 \\
\hline Pathological T stage (T2-3/T1) & 2.093 & $0.717-6.108$ & 0.177 \\
\hline Fuhrman nuclear grade (G3-4/G1-2) & 3.052 & $1.075-8.664$ & 0.036 \\
\hline IL-32 expression (HEG/LEG) & 4.932 & $1.781-13.657$ & 0.009 \\
\hline \multicolumn{4}{|l|}{ Disease-specific survival } \\
\hline ECOG PS $(\geq 1 / 0)$ & 1.244 & $0.384-4.037$ & 0.842 \\
\hline Tumor size $(>10 / \leq 10 \mathrm{~cm})$ & 0.962 & $0.169-5.466$ & 0.965 \\
\hline Pathological T stage (T2-3/T1) & 2.780 & $0.697-11.084$ & 0.147 \\
\hline Fuhrman nuclear grade (G3-4/G1-2) & 4.688 & $1.389-15.814$ & 0.013 \\
\hline IL-32 expression (HEG/LEG) & 6.736 & $2.011-22.563$ & 0.002 \\
\hline \multicolumn{4}{|l|}{ Overall survival } \\
\hline ECOG PS $(\geq 1 / 0)$ & 0.945 & $0.390-2.291$ & 0.901 \\
\hline Tumor size $(>10 / \leq 10 \mathrm{~cm})$ & 0.973 & $0.204-4.647$ & 0.972 \\
\hline Pathological T stage (T2-3/T1) & 1.549 & $0.605-3.964$ & 0.361 \\
\hline Fuhrman nuclear grade (G3-4/G1-2) & 2.741 & $1.056-7.114$ & 0.038 \\
\hline IL-32 expression (HEG/LEG) & 2.992 & $1.066-8.394$ & 0.037 \\
\hline
\end{tabular}

ECOG, Eastern Cooperative Oncology Group; PS, performance status; LEG, low-expression group; HEG, high-expression group. 
carcinoma, have been identified. These factors are significant for appropriate treatment of the patient, providing more information to the patient, and producing treatment plans for new clinical trials. Classically, the prognostic factors of RCC are divided into three categories. Firstly, anatomical prognostic factors include tumor size; extra-renal fat invasion; invasion of main vessels such as the renal vein and inferior vena cava; and lymph node invasion. The tumor size was found to be a significant independent prognostic factor in a number of studies (28); however, there is some controversy in determining the cut-off value for tumor size. Extra-renal fat invasion and direct invasion of the ipsilateral adrenal gland of the RCC are also regarded as prognostic factors (29). Currently, these anatomical prognostic factors are included in the TNM classification as factors of $\mathrm{T}$ staging (30). Nodal invasion was confirmed to be a significant independent prognostic factor (28). However, the sub-classification of nodal invasion by the number of involved nodes is controversial (31). Secondly, histological prognostic factors include Fuhrman nuclear grade; RCC subtype; tumor necrosis; vascular invasion; and the presence of sarcomatoid features. Fuhrman grade is one of the mostly widely accepted prognostic factors, especially in the CCRCC subtype $(32,33)$. CCRCC is more aggressive than papillary and chromophobe types of RCC (34). Patard et al maintain that the histological subtype was a less powerful prognostic factor than the stage of the RCC (35). Tumor necrosis is also a widely accepted prognostic factor in RCC (36). Finally, the clinical prognostic factors include ECOG performance status, patient symptoms, cachexia and anemia (37). Non-classical prognostic factors include certain molecular and genetic markers. The VHL tumor suppressor gene and HIF-1 $\alpha$ are well known prognostic factors in RCC; however, some controversy remains regarding their value as prognostic factors (9). Several studies reported that VEGF and carbonic anhydrase 9 (CAIX) levels also have significance in predicting prognosis. VEGF expression is correlated with aggressive behavior and CAIX correlates with an improved prognosis (5). However, certain studies found no significant relationship between the level of CAIX and a more favorable prognosis (38). The positive reactivity of p53 and elevated serum CRP levels also have value as prognostic factors $(6,8)$. However, the most reliable prognostic factor has not been identified until now.

In this study, we demonstrated the value of IL-32 as a prognostic factor in CCRCC via the immunohistochemical staining of surgically resected human CCRCC tissue. Our data revealed that a high IL-32 expression significantly correlated with the recurrence rate. Additionally, the RFS, DSS and OS rates were significantly decreased in the IL-32 high-expression group compared with the IL-32 low-expression group. Based on these findings, we proposed IL-32 to be a potential prognostic factor for CCRCC. To identify the independent prognostic factors affecting the three different survival rates, univariate and multivariate analyses were performed. The Fuhrman nuclear grade and high IL-32 expression were identified as independent risk factors of RFS, DSS and OS in CCRCC patients. Our results revealed that IL-32 may have novel value as a prognostic factor in localized CCRCC patients undergoing nephrectomy. In our study, other classical prognostic factors of the CCRCC cases, such as tumor size, ECOG PS and pathological staging, did not correlate with the survival rates.
Sorrentino et al, who investigated IL-32 expression in premalignant and malignant lung samples including squamous and non-squamous cell carcinomas, also studied the correlation of IL-32 expression in tumor-infiltrating leukocytes (TIL) and tumor cells (TC) with microvessel density and 5-year survival rates. They found that IL-32 expression in TILs and TCs correlated with high microvessel density in tumors, and the 5-year survival rate was significantly shorter in the IL-32-positive group compared to the IL-32-negative group in all histotypes. These authors suggested that IL-32 expression was beneficial in predicting the prognosis of lung cancer, with the exception of squamous cell carcinoma, since the expression of IL-32 was more significant in metastatic malignancies (18). Results of the present study showed that a high expression of IL-32 in localized CCRCC cases correlated with shorter survival rates compared to cases with a low expression of IL-32. These findings suggest that IL-32 expression in patients with localized CCRCC indicates a poor outcome even after nephrectomy.

The pathogenic mechanism of IL-32 expression in human malignancy is not yet clear; however, certain authors suggested possible activity of IL-32 as a proinflammatory cytokine involved in precancerous inflammation in pancreas and lung cancers $(18,19)$. Recently, Lee et al examined the mechanism by which the high-risk HPV-16 E7 oncogene induced IL-32 expression in cervical cancer cells. These authors investigated IL-32 expression on surgically resected human cervical cancer tissue. Normal cervical epithelium did not express IL-32, as shown by immunohistochemical staining. Their results also showed that COX-2 stimulated IL-32 in response to the HPV-16 E7 oncogene in cervical cancer cells. However, the overexpression of IL-32 inhibited the HPV-16 E7-mediated COX-2 activation pathway and, ultimately, exhibited anti-oncogenic effects (22). In the most recent study on IL-32 and human cancer, Oh et al studied the expression of IL-32 in colon cancer and malignant melanoma cells using xenograft nude mice inoculated with IL-32 $\gamma$-transfected malignant cells (39). They observed the inhibitory effect of IL-32 $\gamma$ on the growth of both malignant cell types. This in vitro study revealed that the inhibitory effect of IL-32 $\gamma$ was due to blockade of the NK- $\mathrm{BB}$ and STAT3 pathways involved in tumor cell growth. Moreover, IL-32 $\gamma$ transgenic mice models showed a decreased expression of antiapoptotic, cell proliferation, and tumor-producing genes such as cleaved caspase-3 and -9, bax, cyclin D, COX-2, and iNOS, whereas the expression of their target apoptotic genes such as BCL-2 and X-chromosome inhibitor of apoptosis protein was significantly increased (39). The findings of the two recent studies indicate that IL-32 has anticancer effects in human malignancies. In our study, CCRCC cases that stained strongly for IL-32 exhibited more aggressive behavior. As a result, IL-32 expression in surgically resected human malignancy tissue exhibits a potentially reactive host-immune response to the malignant cells. Thus, IL-32 expression was more intense in the more aggressive cases. Further studies are required to increase understanding of the role of IL-32 in carcinogenesis and cancer progression.

In conclusion, our findings indicate that IL-32 overexpression in localized CCRCC is a novel prognostic factor in surgically treated patients. Clinically, the analyses of IL-32 expression in surgically resected or biopsied specimens may 
aid the determination of future treatment plans or prediction of patient prognosis. Additionally, further investigations are required to increase understanding of the pathogenesis of IL-32 in human cancers.

\section{Acknowledgements}

This study was supported by a National Research Foundation of Korea (NRF) grant funded by the Korea government (MEST) (No. 2011-0006229) and by a grant from the National R\&D Program for Cancer Control Ministry of Health and Welfare, Republic of Korea (No. 0720560).

\section{References}

1. Jemal A, Siegel $\mathrm{R}, \mathrm{Xu} \mathrm{J}$ and Ward E: Cancer statistics CA Cancer J Clin 60: 277-300, 2010.

2. Zisman A, Pantuck AJ, Wieder J, et al: Risk group assessment and clinical outcome algorithm to predict the natural history of patients with surgically resected renal cell carcinoma. J Clin Oncol 20: 4559-4566, 2002.

3. Motzer RJ, Hutson TE, Tomczak P, et al: Overall survival and updated results for sunitinib compared with interferon alfa in patients with metastatic renal cell carcinoma. J Clin Oncol 27: 3584-3590, 2009.

4. Finley DS, Pantuck AJ and Belldegrun AS: Tumor biology and prognostic factors in renal cell carcinoma. Oncologist 16 Suppl 2: 4-13, 2011.

5. Phuoc NB, Ehara H, Gotoh T, et al: Prognostic value of the co-expression of carbonic anhydrase IX and vascular endothelial growth factor in patients with clear cell renal cell carcinoma. Oncol Rep 20: 525-530, 2008.

6. Girgin C, Tarhan H, Hekimgil M, Sezer A and Gurel G: P53 mutations and other prognostic factors of renal cell carcinoma. Urol Int 66: 78-83, 2001.

7. Klatte T, Seligson DB, Riggs SB, et al: Hypoxia-inducible factor 1 alpha in clear cell renal cell carcinoma. Clin Cancer Res 13: 7388-7393, 2007.

8. Cho DS, Kim SJ, Lee SH, Ahn HS, Kim YS and Kim SI: Prognostic significance of preoperative C-reactive protein elevation and thrombocytosis in patients with non-metastatic renal cell carcinoma. Korean J Urol 52: 104-109, 2011.

9. Yao M, Yoshida M, Kishida T, et al: VHL tumor suppressor gene alterations associated with good prognosis in sporadic clear-cell renal carcinoma. J Natl Cancer Inst 94: 1569-1575, 2002.

10. Dahl CA, Schall RP, He HL and Cairns JS: Identification of a novel gene expressed in activated natural killer cells and T cells J Immunol 148: 597-603, 1992.

11. Kim SH, Han SY, Azam T, Yoon DY and Dinarello CA: Interleukin-32: A cytokine and inducer of TNF alpha. Immunity 22: 131-142, 2005.

12. Chen Q, Carroll HP and Gadina M: The newest interleukins: recent additions to the ever-growing cytokine family. Vitam Horm 74: 207-228, 2006.

13. Csernok E, Holle JU and Gross WL: Proteinase 3, protease-activated receptor-2 and interleukin-32: linking innate and autoimmunity in Wegener's granulomatosis. Clin Exp Rheumatol 26: S112-S117, 2008.

14. Joosten LA, Netea MG, Kim SH, et al: IL-32, a proinflammatory cytokine in rheumatoid arthritis. Proc Natl Acad Sci USA 103 3298-3303, 2006.

15. Netea MG, Azam T, Lewis EC, et al: Mycobacterium tuberculosis induces interleukin-32 production through a caspase-1/ IL-18/interferon-gamma-dependent mechanism. PLoS Med 3: e277, 2006

16. Shioya M, Nishida A, Yagi Y, et al: Epithelial overexpression of interleukin-32alpha in inflammatory bowel disease. Clin Exp Immunol 149: 480-486, 2007.

17. Seo EH, Kang J, Kim KH, et al: Detection of expressed IL-32 in human stomach cancer using ELISA and immunostaining. J Microbiol Biotechnol 18: 1606-1612, 2008.

18. Sorrentino C and Di Carlo E: Expression of IL-32 in human lung cancer is related to the histotype and metastatic phenotype. Am J Respir Crit Care Med 180: 769-779, 2009.
19. Nishida A, Andoh A, Inatomi O and Fujiyama Y: Interleukin-32 expression in the pancreas. J Biol Chem 284: 17868-17876, 2009.

20. Marcondes AM, Mhyre AJ, Stirewalt DL, Kim SH, Dinarello CA and Deeg HJ: Dysregulation of IL-32 in myelodysplastic syndrome and chronic myelomonocytic leukemia modulates apoptosis and impairs NK function. Proc Natl Acad Sci USA 105: 2865-2870, 2008.

21. Ko NY, Chang SH, Lee JH, et al: Unique expression of a small IL-32 protein in the Jurkat leukemic T cell line. Cytokine 42: 121-127, 2008

22. Lee S, Kim JH, Kim H, et al: Activation of the interleukin-32 pro-inflammatory pathway in response to human papillomavirus infection and over-expression of interleukin-32 controls the expression of the human papillomavirus oncogene. Immunology 132: 410-420, 2011.

23. Carter KL, Cahir-McFarland E and Kieff E: Epstein-Barr virus-induced changes in B-lymphocyte gene expression. J Virol 76: 10427-10436, 2002.

24. Park GH, Choe J, Choo HJ, Park YG, Sohn J and Kim M: Genome-wide expression profiling of 8-chloroadenosine-and 8-chloro-cAMP-treated human neuroblastoma cells using radioactive human cDNA microarray. Exp Mol Med 34: 184-193, 2002.

25. Goda C, Kanaji T, Kanaji S, et al: Involvement of IL-32 in activation-induced cell death in T cells. Int Immunol 18: 233-240, 2006.

26. Netea MG, Azam T, Ferwerda G, et al: IL-32 synergizes with nucleotide oligomerization domain (NOD) 1 and NOD2 ligands for IL-1beta and IL- 6 production through a caspase 1-dependent mechanism. Proc Natl Acad Sci USA 102: 16309-16314, 2005.

27. Klatte T, Pantuck AJ, Kleid MD and Belldegrun AS: Understanding the natural biology of kidney cancer: implications for targeted cancer therapy. Rev Urol 9: 47-56, 2007.

28. Delahunt B, Kittelson JM, McCredie MR, Reeve AE, Stewart JH and Bilous AM: Prognostic importance of tumor size for localized conventional (clear cell) renal cell carcinoma: assessment of TNM T1 and T2 tumor categories and comparison with other prognostic parameters. Cancer 94: 658-664, 2002.

29. Terrone C, Gontero P, Volpe A, et al: Proposal of an improved prognostic classification for pT3 renal cell carcinoma. J Urol 180: 72-78, 2008.

30. Eble JN, Sauter G, Ebstein J and Sesterhenn I: Pathology and Genetics of Tumours of the Urinary System and Male Genital Organs. IARC-Press, Lyon, 2004.

31. Ficarra V, Schips L, Guille F, et al: Multiinstitutional European validation of the 2002 TNM staging system in conventional and papillary localized renal cell carcinoma. Cancer 104: 968-974, 2005.

32. Fuhrman SA, Lasky LC and Limas C: Prognostic significance of morphologic parameters in renal cell carcinoma. Am J Surg Pathol 6: 655-663, 1982.

33. Delahunt B, Sika-Paotonu D, Bethwaite PB, et al: Fuhrman grading is not appropriate for chromophobe renal cell carcinoma. Am J Surg Pathol 31: 957-960, 2007.

34. Cheville JC, Lohse CM, Zincke H, Weaver AL and Blute ML: Comparisons of outcome and prognostic features among histologic subtypes of renal cell carcinoma. Am J Surg Pathol 27: 612-624, 2003

35. Patard JJ, Leray E, Rioux-Leclercq N, et al: Prognostic value of histologic subtypes in renal cell carcinoma: a multicenter experience. J Clin Oncol 23: 2763-2771, 2005.

36. Lee SE, Byun SS, Oh JK, et al: Significance of macroscopic tumor necrosis as a prognostic indicator for renal cell carcinoma. J Urol 176: 1332-1337, 2006.

37. Kim HL, Belldegrun AS, Freitas DG, et al: Paraneoplastic signs and symptoms of renal cell carcinoma: implications for prognosis. J Urol 170: 1742-1746, 2003.

38. Leibovich BC, Sheinin Y, Lohse CM, et al: Carbonic anhydrase IX is not an independent predictor of outcome for patients with clear cell renal cell carcinoma. J Clin Oncol 25: 4757-4764, 2007.

39. Oh J, Cho M, Kim J, et al: IL-32 $\gamma$ inhibits cancer cell growth through inactivation of NF- $\kappa \mathrm{B}$ and STAT3 signals. Oncogene, 2011 (E-pub ahead of print). 